\title{
Spectophotometric method for determination of certain cephalosporins using 4-chloro-7-nitrobenzo-2-oxa-1, 3-diazole (NBD-Cl)
}

\author{
Azza H. Rageh*, Salwa R. El-Shaboury, Gamal A. Saleh, Fardous A. Mohamed
}

Department of Pharmaceutical Analytical Chemistry, Facutly of Pharmacy, Assiut University, Assiut, Egypt; *Corresponding Author: azhesham@yahoo.com

Received 11 February 2010; revised 23 March 2010; accepted 30 March 2010.

\begin{abstract}
A simple, accurate and precise spectrophotometric method has been proposed for the determination of eleven cephalosporins, namely; cefaclor monohydrate, cefadroxil monohydrate, cefalexin anhydrous, cefradine anhydrous, cefotaxime sodium, cefoperazone sodium, ceftriaxone sodium, ceftazidime penthydrate, cefazolin sodium, cefixime and cefpodoxime proxetil in bulk drug and in pharmaceutical formulations. The method depends on hydrolysis of the studied drugs using $0.5 \mathrm{M} \mathrm{NaOH}$ at $100^{\circ} \mathrm{C}$ and subsequent reaction of the formed sulfide ions with NBD-Cl (4-chloro-7-nitrobenzo-2-oxa-1, 3-diazole) to form a yellow-colored chromogen measured at $390 \mathrm{~nm}$. Different variables affecting the reaction (e.g. $\mathrm{NaOH}$ concentration, hydrolysis time, NBD-Cl concentration and diluting solvent) were studied and optimized. Under the optimum conditions, linear relationships with good correlation coefficients (0.99900.9999 ) were found in the range of $5-160 \mu \mathrm{g} \mathrm{mL}^{-1}$ for all studied drugs. The limits of assay detection and quantitiation ranged from 0.289 to 5.867 and from 0.878 to $17.778 \mu \mathrm{g} \mathrm{mL}^{-1}$; respectively. The accuracy and precision of the proposed method were satisfactory. The method was successfully applied for analysis of the studied drugs in their pharmaceutical formulations and the recovery percentages ranged from 96.6 to $103.5 \%$.
\end{abstract}

Keywords: Spectrophotometry; Cephalosporins; NBD-Cl; Pharmaceutical Analysis

\section{INTRODUCTION}

Cephalosporins have been used since 1948. These anti- biotics have assumed a prominent role in modern antimicrobial therapy due to enhanced intrinsic microbiological activities and favorable safety profile. Chemical structures of cephalosporins drive from the 7-aminocephalosporanic acid (7-ACA) composed of a $\beta$-lactam ring fused with a dihydrothaizine ring (Figure 1), but differ in the nature of substituents at the 3- and/or 7-positions of the cephem ring. These substituents affect either the pharmacokinetic properties (3-position) or the antibacterial spectrum (7-position) of the cephalosporins $[1,2]$. Traditionally, cephalosporins are divided into first-, second-, third-, and fourth-generation agents. Table 1 shows cephalosporins studied in this work. Several methods have been reported for cephalosporins determination. The official procedures in pharmaceutical preparations utilize high-performance liquid chromatography (HPLC) [3] which is expensive. Other reported procedures include spectrophotometric [4-9], spectrofluorimetric [10-13], chemiluminescence [14-16], chromatographic [17-20] and electrochemical methods [21-24] and most of them are lengthy and/or tedious.

The hydrolytic degradation of cephalosporins was very often used as a preliminary step in the analytical procedure used for their determinations [25-32]. The literature reveals that many spectrophotometric methods were developed for cephalosporins determinations that based on hydrolysis of these drugs using alkaline degra-

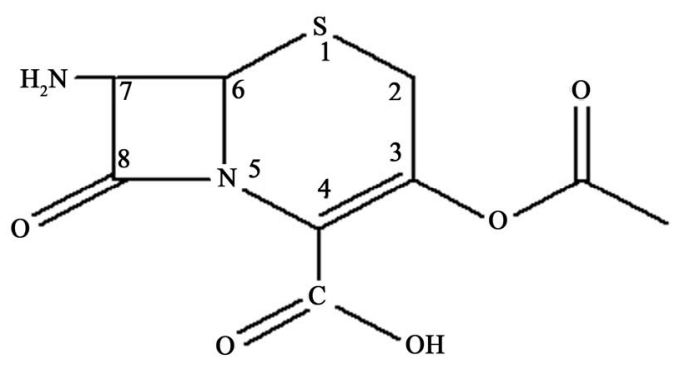

Figure 1. Chemical structure of 7-aminocephalosporanic acid. 
Table 1. Chemical structures of the investigated cephalosporin antibiotics.

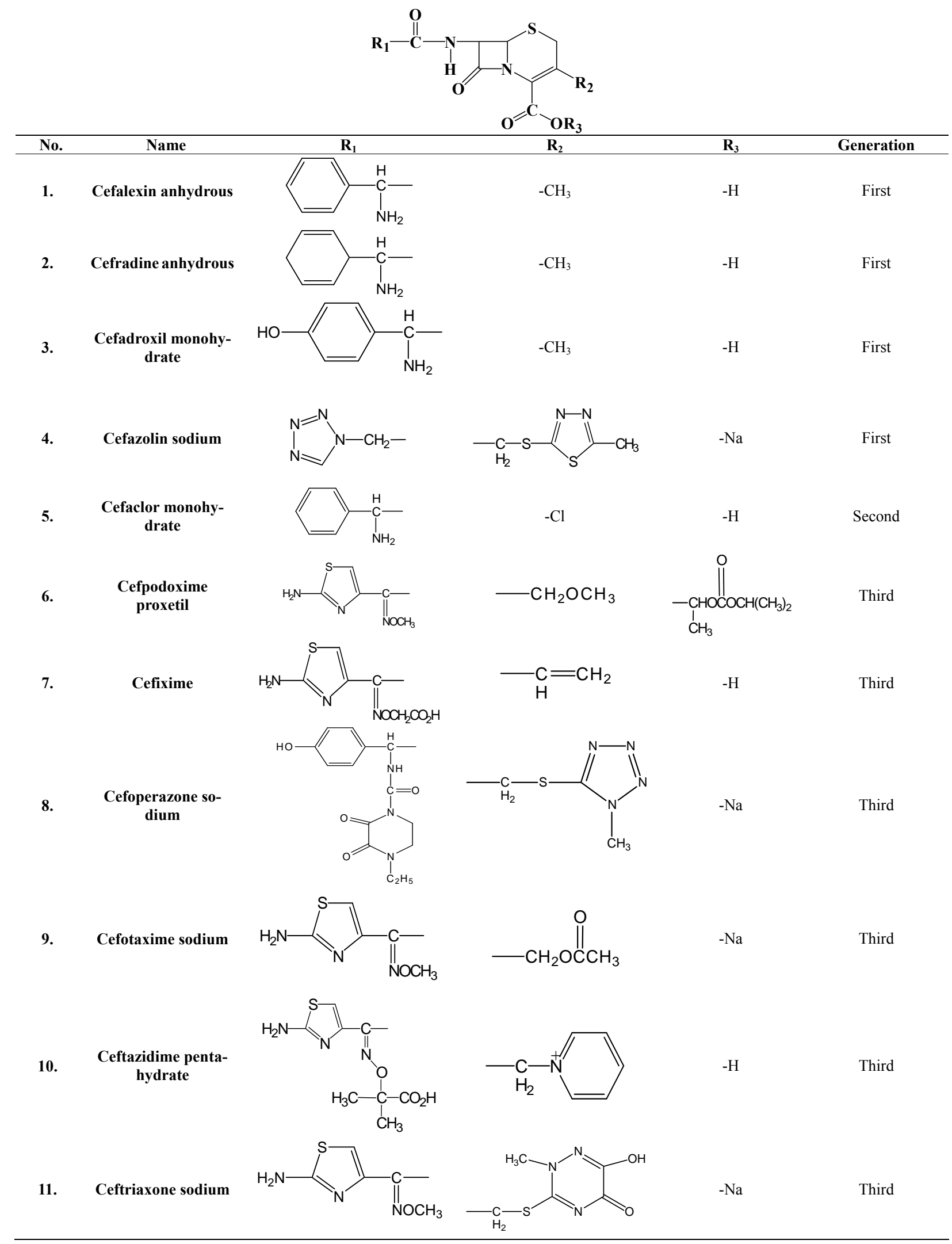


dation and subsequent reaction of the formed sulfide ions with chromogenic reagents [26,27].

NBD-Cl (Figure 2) has been reported as fluorogenic reagent for determination of amines [33] and for spectrophotometric determination of many compounds [34-41]. Thiocompounds have been reported to form intensely colored products in an alkaline medium with NBD-Cl which could be used for their colorimetric determination [42]. It is always required to develop analytical methods using low cost techniques. UV-Vis spectrophotometry is still considered a convenient and economical technique for routine analysis of drugs in pharmaceutical formulations. On the basis of the aforementioned reasons, it was decided to develop a quantitative method for the determination of the studied cephalosporins based on their alkaline hydrolysis and subsequent reaction of the resulting hydrolysates with NBD-Cl, which may be used for their analysis either in pure forms or in pharmaceutical formulations. This method is selective for cephalosporins, since other $\beta$-lactam antibiotics such as penicillins do not give sulfide ions under the degradation conditions employed [27,43-45].

\section{EXPERIMENTAL}

\subsection{Apparatus}

Shimadzu UV-1700 PC, UV-Visible Spectrophotometer (Tokyo, Japan), ultrasonic cleaner (Cole-Parmer, Chicago, USA), sartorious handy balance-H51 (Hannover, Germany) and MLV type thermostatically controlled water bath (Salvis AG Emmenbruck, Luzern, Germany).

\subsection{Materials and Reagents}

All solvents used were of analytical-reagent grade, sodium hydroxide (El-Nasr Chemical Co. Cairo, Egypt) $0.5 \mathrm{M}$ aqueous solution, hydrochloric acid (El-Nasr Chemical Co. Cairo, Egypt), 4-cholor-7-nitrobenzofurazan [NBD-Cl] (Fluka Chemie AG, Switzerland) freshly prepared $\left(3 \times 10^{-3} \mathrm{M}\right)$ equivalent to $0.060 \% \mathrm{w} / \mathrm{v}$ in acetone, samples of cephalosporins were generously supplied by their respective manufacturers and were used as supplied: cefaclor monohydrate and cefradine

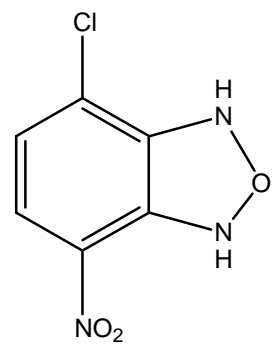

Figure 2. Chemical structure of NBD-Cl. anhydrous (Sigma Chemical Co., St. Louis, USA), cefadroxil monohydrate (Amoun Pharmaceutical Industries Co., APIC, Cairo, Egypt), cefalexin anhydrous (GalaxoWellcome, S.A.E., El Salam City, Cairo, Egypt), cefotaxime sodium (CID, Cairo, Egypt), cefoperazone sodium (Pfizer Co., Egypt), ceftazidime pentahydrate and ceftriaxone sodium (T3A Pharma Group, Assiut, Egypt), cefpodoxime proxetil (Hoechst Marion Roussel, S. A. E., Cairo, Egypt), cefixime (El-Hekma Co., Cairo, Egypt) and cefazolin sodium (Bristol-Myers Squibb Pharmaceutical Co., Cairo, Egypt) and pharmaceutical formulations containing the studied drugs were purchased from local market.

\subsection{Preparation of Standard Solutions}

Stock solutions containing $100 \mathrm{mg} \mathrm{mL}^{-1}$ of each cephalosporin were prepared in double distilled water (methanol was used in case of cefadroxil monohydrate, cefalexin anhydrous, cefaclor monohydrate, cefradine anhydrous, cepodoxime proxetil and cefixime). Working standard solutions containing $0.5-2.5 \mathrm{mg} \mathrm{mL}^{-1}$ (in case of cefadroxil monohydrate and cefalexin anhydrous), 1$6 \mathrm{mg} \mathrm{mL}^{-1}$ (in case of cefradine anhydrous), $2-8 \mathrm{mg} \mathrm{mL}^{-1}$ (in case of cefaclor monohydrate, cefazolin sodium, cefotaxime sodium, ceftriaxone sodium and cefpodoxime proxetil), 2-10 $\mathrm{mg} \mathrm{mL}^{-1}$ (in case of cefixime) and 2$16 \mathrm{mg} \mathrm{mL}^{-1}$ (in case of cefoperazone sodium and ceftazidime pentahydrate) were prepared by suitable dilution of the stock solution with double distilled water (in case of cefadroxil monohydrate, cefalexin anhydrous, cefaclor monohydrate, cepodoxime proxetil and cefixime, dilution was made using methanol). The stock and working standard solutions must be freshly prepared.

\subsection{Preparation of Sample Solutions}

Tablets and capsules. Twenty tablets or the contents of 20 capsules were weighed, finely powdered and mixed thoroughly. An accurately weighed amount of the powder obtained from tablets or capsules equivalent to $250 \mathrm{mg}$ of each drug was transferred into a $25-\mathrm{mL}$ volumetric flask, dissolved in about $10 \mathrm{~mL}$ double distilled water (10 mL methanol was used in case of cefadroxil monohydrate, cefalexin anhydrous, cefaclor monohydrate, cefradine anhydrous, cepodoxime proxetil and cefixime), sonicated for $15 \mathrm{~min}$, diluted to the mark with double distilled water (in case of cefadroxil monohydrate, cefalexin anhydrous, cefaclor monohydrate, cefradine anhydrous, cefpodoxime proxetil and cefixime, dilution was made using methanol), mixed well and filtered; the first portion of the filtrate was rejected. Further dilutions with the same solvent were made to obtain sample solution containing the specified concentration for each drug 
as mentioned under the preparation of standard solutions and then the general procedure was followed.

Vials and powder for oral suspension. An accurately weighed amount of powder equivalent to $250 \mathrm{mg}$ of each drug was transferred into a $25-\mathrm{mL}$ volumetric flask, then the procedure was followed as under tablets and capsules beginning from (dissolved in about $10 \mathrm{~mL}$ double distilled water..........).

\subsection{General Procedure}

Accurately measured one milliliter aliquot volume of the standard or sample solutions was transferred into $10-\mathrm{mL}$ volumetric flask. $5 \mathrm{~mL}$ of $0.5 \mathrm{M} \mathrm{NaOH}$ were added and the flask was heated in a boiling water-bath for $30 \mathrm{~min}$, cooled to room temperature and completed to volume with double distilled water. One milliliter of the resulting drug hydrolysate was pipetted into $10-\mathrm{mL}$ volumetric flask, $1.0 \mathrm{~mL}$ of $3 \times 10^{-3} \mathrm{M}$ NBD-Cl was added followed by $1 \mathrm{~mL}$ of concentrated $\mathrm{HCl}$. The resulting solution was mixed well and the flask was completed to volume with ethanol. The absorbance was measured at $390 \mathrm{~nm}$ against reagent blank treated similarly.

\section{RESULTS AND DISCUSSION}

\subsection{Absorption Spectra}

As shown in Figure 3, the absorption spectrum of $\mathrm{NBD}-\mathrm{Cl}$ in acetone shows a maximum absorption at $340 \mathrm{~nm}$. All the investigated drugs after alkaline hydrolysis give a very weak absorption taking cefalexin anhydrous hydrolysate as a representative example which gives a very broad absorption maximum at $350 \mathrm{~nm}$. The interaction colored product of cefalexin anhydrous hydrolysate with NBD-Cl shows absorption maximum at $390 \mathrm{~nm}$ (Figure 3).

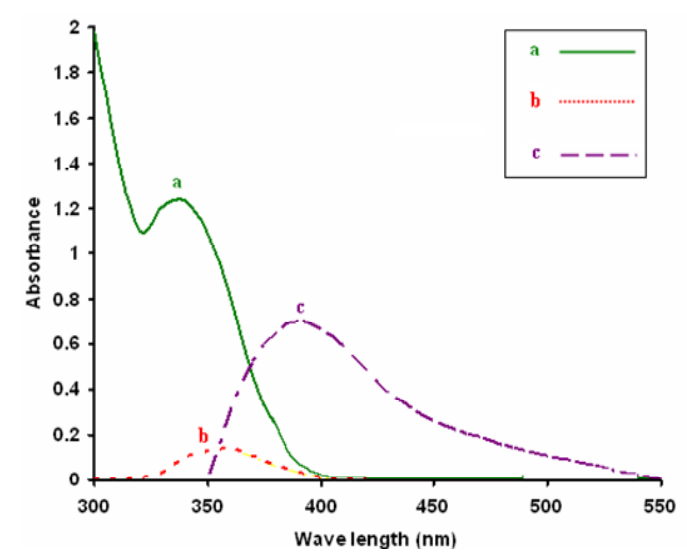

Figure 3. Absorption spectra of (a) NBD-Cl $(3 \times$ $\left.10^{-3} \mathrm{M}\right)$, (b) cefalexin anhydrous hydrolysate alone (20 $\mu \mathrm{g} \mathrm{mL}^{-1}$ ) and (c) the reaction colored product between NBD-Cl and cefalexin anhydrous hydrolysate.

\subsection{Optimization of Reaction Variables}

Since the developed method depends on the formation of colored product by the interaction of NBD-Cl with sulfide ions resulted from the alkaline degradation of cephalosporins so, optimization studies were carried out extensively to find the optimum conditions for the alkaline degradation and subsequently the optimum yield of sulfide ions and the maximum stability of the chromogen formed taking cefalexin anhydrous $\left(15 \mu \mathrm{g} \mathrm{mL} \mathrm{m}^{-1}\right)$ as a representative example for these studies. These variables include:

Effect of $\mathrm{NaOH}$ concentration. The influence of sodium hydroxide concentration on producing the maximum absorption intensity was investigated using 0.1 1.0 $\mathrm{M} \mathrm{NaOH}$ keeping other factors constant. Maximum absorption readings were obtained upon using $0.5 \mathrm{M}$ $\mathrm{NaOH}$; above this concentration and up to $1 \mathrm{M} \mathrm{NaOH}$, the absorbance remains constant. So, this concentration was selected for further work (Figure 4).

Effect of hydrolysis time. The effect of hydrolysis time on the absorption intensity was studied using different heating times in a boiling water bath (at $100^{\circ} \mathrm{C}$ ) starting from $10 \mathrm{~min}$ until 2 hours and the reaction was carried out as usual. The obtained absorbance readings were plotted against hydrolysis time. The maximum absorption intensity was attained after $20 \mathrm{~min}$ and remained stable for at least $100 \mathrm{~min}$. Thirty minutes hydrolysis time was used in all subsequent experiments as shown in Figure 5.

Effect of NBD-Cl concentration. The concentration of NBD-Cl, for the maximum color development was varied in the range of $0.75 \times 10^{-3}-4 \times 10^{-3} \mathrm{M}$. It was found that $1 \mathrm{~mL}$ of $3 \times 10^{-3} \mathrm{MNBD}-\mathrm{Cl}$ was the most suitable con-

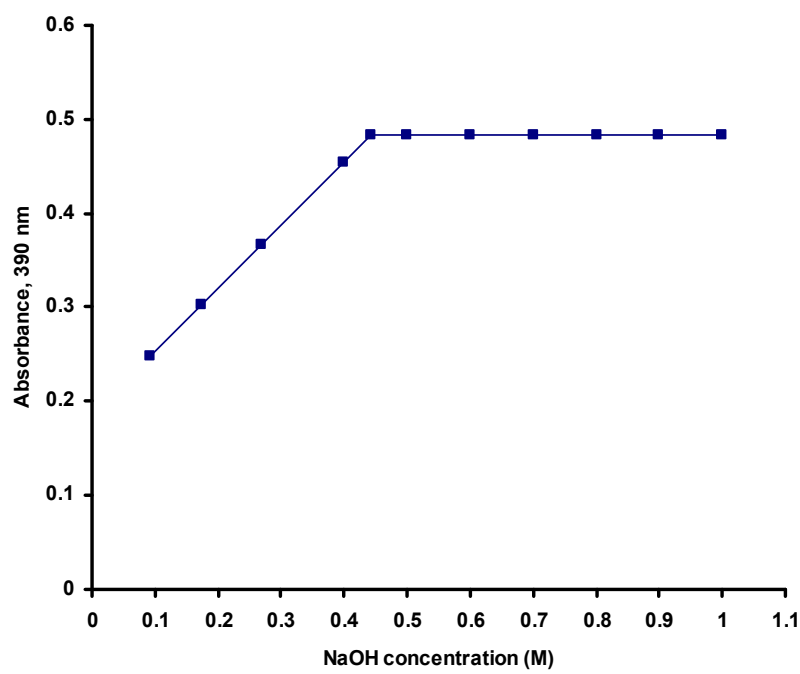

Figure 4. Effect of $\mathrm{NaOH}$ concentration on the absorbance of the reaction colored product at $390 \mathrm{~nm}$. 


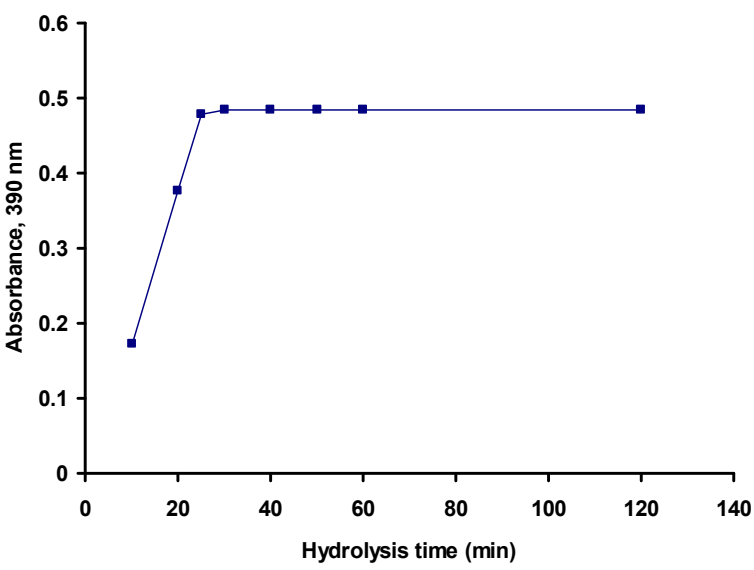

Figure 5. Effect of hydrolysis time on the absorbance of the reaction colored product at $390 \mathrm{~nm}$.

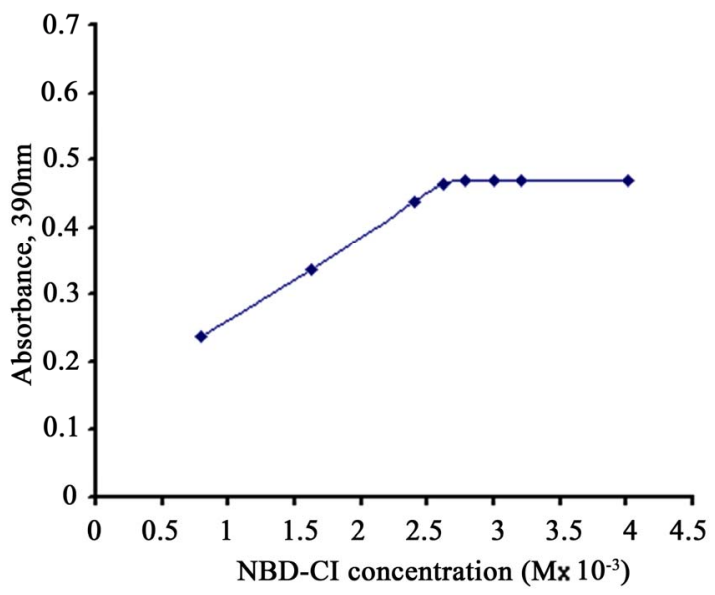

Figure 6. Effect of NBD-Cl concentration on the absorbance of the reaction colored product at $390 \mathrm{~nm}$.

centration for determination of the studied drugs as shown in Figure 6. Owing to the presence of labile chloride, a daily fresh solution is recommended.

Effect of type and concentration of acid. Different acids such as sulfuric, hydrochloric, perchloric, nitric and acetic acids were tested to determine the most suitable acid for the reaction. One milliliter of concentrated hydrochloric acid was selected in this study as it gave the highest absorbance readings taking cefalexin anhydrous $\left(15 \mu \mathrm{g} \mathrm{mL}^{-1}\right)$ as a representative example (Table 2).

Further investigations were carried out in order to find the most suitable concentration of hydrochloric acid. It was observed that higher absorbance readings and more reproducible results were obtained upon increasing hydrochloric acid concentration. As a result of these investigations, $1 \mathrm{~mL}$ of concentrated hydrochloric acid was used for subsequent work.

Effect of reaction time. The reaction between the investigated drugs hydrolysates and $\mathrm{NBD}-\mathrm{Cl}$ was very
Table 2. Effect of different acids on the absorbance readings of the reaction colored product of cefalexin anhydrous ${ }^{\mathbf{a}}$ with NBD-Cl.

\begin{tabular}{lc}
\hline Acid (1 mL) & Absorbance $^{\mathbf{b}}$ \\
\hline Hydrochloric acid & 0.460 \\
Sulfuric acid & 0.400 \\
Perchloric acid & 0.413 \\
Acetic acid & 0.210 \\
Nitric acid & 0.315 \\
\hline${ }^{\text {a}}$ Cefalexin anhydrous concentration used is $15 \mu \mathrm{g} \mathrm{mL}{ }^{-1}$; ${ }^{\mathbf{b}}$ Average \\
of three determinations.
\end{tabular}

rapid and the interaction colored product can survive before dilution unchanged for at least 1 hour. However, measurements were achieved instantaneously.

Effect of diluting solvent. Different solvents were tested in order to select the most appropriate solvent for optimum color development. The results given in Table 3 show small shifts in the position of the maximum absorption peak. The absorption intensities were slightly influenced. Ethanol was used throughout this work because it gave the highest absorbance readings and the most reproducible results.

Stability of the reaction colored product. Stability time was obtained by following the absorbance readings of the developed reaction product for 24 hours at room temperature $\left(25 \pm 5^{\circ} \mathrm{C}\right)$. It was found that the produced color was stable for 24 hours for all studied drugs.

\subsection{Calibration Curves}

Linear relationship was obtained for all studied drugs by applying the developed method (Table 4). Good linearity of the calibration curves were clearly evident by excellent correlation coefficients which ranged from 0.9990 to 0.9999 and coefficients of determination ranged from 0.9978 to 0.9998 . This wide variation in the linearity range may be attributed to the different yields

Table 3. Effect of solvent on $\lambda_{\max }$ and the absorbance of the formed chromogen between cefalexin anhydrous ${ }^{\mathrm{a}}$ and NBD-Cl.

\begin{tabular}{lll}
\hline Solvent & $\lambda_{\max }(\mathrm{nm})$ & $\mathrm{A}^{\mathrm{b}}$ \\
\hline Water & 404 & 0.404 \\
Ethanol & 390 & 0.470 \\
Methanol & 391 & 0.401 \\
Acetone & 392 & 0.427 \\
Acetonitrile & 398 & 0.456 \\
Propan-1-ol & 390 & 0.467 \\
Propan-2-ol & 389 & 0.463 \\
Dimethylformamaide & 393 & 0.425 \\
Dimehtylsulfoxide & 401 & 0.458 \\
\hline
\end{tabular}

${ }^{\mathrm{a}}$ Cefalexin anhydrous concentration is $15 \mu \mathrm{g} \mathrm{mL}{ }^{-1}$; ${ }^{\mathbf{b}}$ Average of 3 determinations. 
Table 4. Summary of quantitative parameters and statistical data using the proposed procedure.

\begin{tabular}{|c|c|c|c|c|c|c|c|}
\hline Drug & $\begin{array}{l}\text { Intercept } \\
\text { (a) } \pm \mathbf{S D}^{\mathbf{a}}\end{array}$ & $\begin{array}{c}\text { Slope } \\
\text { (b) } \pm \text { SD }^{\mathbf{a}}\end{array}$ & $\begin{array}{c}\text { Linear- } \\
\text { ity } \\
\text { Range } \\
(\mu \mathrm{g} \\
\left.\mathrm{mL}^{-1}\right) \\
\end{array}$ & $\begin{array}{l}\text { Correlation } \\
\text { coefficient } \\
\text { (r) }\end{array}$ & $\begin{array}{c}\text { Determina- } \\
\text { tion coeffi- } \\
\text { cient } \\
\left(\mathbf{r}^{2}\right)\end{array}$ & $\begin{array}{c}\mathbf{L O D}^{\mathrm{b}} \\
(\mu \mathrm{g} \\
\left.\mathbf{m L} \mathbf{L}^{-1}\right)\end{array}$ & $\begin{array}{c}\mathbf{L O Q}^{\mathrm{c}} \\
(\mu \mathrm{g} \\
\left.\mathrm{mL}^{-1}\right)\end{array}$ \\
\hline $\begin{array}{l}\text { Cefadroxil monhy- } \\
\text { drate }\end{array}$ & $-0.013 \pm\left(3.6 \times 10^{-3}\right)$ & $0.041 \pm\left(0.2 \times 10^{-3}\right)$ & $5-25$ & 0.9999 & 0.9998 & 0.29 & 0.88 \\
\hline Cefalexin anhydrous & $-0.126 \pm\left(5.0 \times 10^{-2}\right)$ & $0.398 \pm\left(1.2 \times 10^{-3}\right)$ & $5-25$ & 0.9999 & 0.9998 & 0.42 & 1.26 \\
\hline Cefradine anhydrous & $0.076 \pm\left(7.5 \times 10^{-3}\right)$ & $0.013 \pm\left(1.5 \times 10^{-4}\right)$ & $10-60$ & 0.9999 & 0.9998 & 1.90 & 5.77 \\
\hline $\begin{array}{l}\text { Cefaclor monohy- } \\
\text { drate }\end{array}$ & $0.055 \pm\left(1.3 \times 10^{-2}\right)$ & $0.010 \pm\left(2.1 \times 10^{-4}\right)$ & $20-80$ & 0.9996 & 0.9992 & 4.29 & 13.00 \\
\hline Cefazolin sodium & $0.016 \pm\left(4.3 \times 10^{-3}\right)$ & $0.009 \pm\left(1.9 \times 10^{-4}\right)$ & $20-80$ & 0.9994 & 0.9988 & 1.58 & 4.78 \\
\hline Ceftriaxone sodium & $0.033 \pm\left(5.3 \times 10^{-3}\right)$ & $0.010 \pm\left(0.5 \times 10^{-4}\right)$ & $20-80$ & 0.9996 & 0.9992 & 1.75 & 5.30 \\
\hline Cefotaxime sodium & $0.056 \pm\left(1.2 \times 10^{-2}\right)$ & $0.010 \pm\left(1.5 \times 10^{-4}\right)$ & $20-80$ & 0.9990 & 0.9980 & 3.96 & 12.00 \\
\hline Cefpodoxime proxetil & $0.046 \pm\left(1.6 \times 10^{-2}\right)$ & $0.009 \pm\left(1.3 \times 10^{-4}\right)$ & $20-80$ & 0.9990 & 0.9980 & 5.87 & 17.78 \\
\hline Cefixime & $0.095 \pm\left(3.2 \times 10^{-3}\right)$ & $0.007 \pm\left(0.2 \times 10^{-4}\right)$ & $20-100$ & 0.9989 & 0.9978 & 1.51 & 4.57 \\
\hline Cefoperazone sodium & $0.019 \pm\left(8.3 \times 10^{-3}\right)$ & $0.005 \pm\left(0.6 \times 10^{-4}\right)$ & $20-160$ & 0.9998 & 0.9996 & 5.48 & 16.60 \\
\hline $\begin{array}{l}\text { Ceftazidime penta- } \\
\text { hydrate }\end{array}$ & $0.048 \pm\left(7.4 \times 10^{-3}\right)$ & $0.005 \pm\left(0.7 \times 10^{-4}\right)$ & $20-160$ & 0.9994 & 0.9988 & 4.88 & 14.80 \\
\hline
\end{tabular}

of sulfide ions from the studied cephalosporins [45].

\subsection{Method Validation Study}

The method was validated according ICH guidelines on the validation of analytical methods [46] and complied with USP 31 validation guidelines [3]. All results were expressed as percentages, where $n$ represents the number of values. For the statistical analysis Excel 2003 (Microsoft Office) was used. A 5\% significance level was selected.

$L O D$ and $L O Q$. The limits of detection and quantitation for all studied drugs ranged from 0.29 to 5.87 and from 0.88 to $17.78 \mu \mathrm{g} \mathrm{mL}^{-1}$; respectively which indicate high sensitivity of the proposed method (Table 4).

Accuracy. The accuracy of the method was determined by investigating the recovery of each of the studied drugs at three concentration levels covering the specified range (six replicates of each concentration). The results shown in Table 5 depict good accuracy and recovery percentage ranged from 98.0 to $102.3 \%$.

Precision. As shown in Table 6, the small values of SD and \% RSD point to high precision of the proposed method.

Selectivity. The effect of the presence of common excipients such as; starch, talc, lactose, glucose, sucrose, magnesium-stearate and gum acacia was studied. It was found that no interference was introduced by any of them.

Robustness. Robustness was examined by evaluating the influence of small variation in the experimental parameters on the analytical performance of the proposed method [47]. The studied parameters were: $\mathrm{NaOH}$ con- centration, NBD-Cl concentration, heating temperature and heating time on the method suitability and sensitivity. It was found that none of these variables significantly affects the performance of the method (Table 7) which indicates the robustness of the proposed method.

\subsection{Applications to the Analysis of Pharmaceutical Dosage Forms}

The proposed method was applied successfully for determination of the studied drugs in their pharmaceutical dosage forms. Six replicate measurements were made in each case, the results obtained were validated by comparison with a previously reported method [48]. No significant difference was found by applying $t$ - and $F$-tests at $95 \%$ confidence level indicating good accuracy and precision (Table 8). Recovery studies were also carried out by standard addition method [49]. The results in Table 9 indicate good recoveries (96.0 to $103.8 \%$ ) and confirm that there is no interference from frequently encountered excipients or additives.

\subsection{Suggested Reaction Mechanism}

Cephalosporins were previously reported to produce sulfide ions upon alkaline degradation and it was found to be one of their major degradation products [43-45, 50-55]. NBD-Cl is an active halide derivative, which was considered as a likely target for good nuclophiles, under alkaline conditions, such as amines, amino acids and thiocompounds [40-42].

In the proposed method, sulfide ions were allowed to react with NBD-Cl via $\mathrm{SN}_{2}$ mechanism. The high nucleophilicity of sulfide ions, the presence of $\mathrm{Cl}^{-}$anion as 
Table 5. Accuracy of the proposed method for analysis of the studied drugs at three concentration levels.

\begin{tabular}{|c|c|c|c|}
\hline \multirow[b]{2}{*}{ Drug } & \multicolumn{3}{|c|}{ Recovery $(\%) \pm$ SD $^{\mathbf{a}}$} \\
\hline & $25 \mu \mathrm{g} \mathrm{mL} \mathrm{L}^{-1}$ & $50 \mu \mathrm{g} \mathrm{mL} \mathrm{L}^{-1}$ & $75 \mu \mathrm{g} \mathrm{mL}^{-1}$ \\
\hline Cefaclor monohydrate & $100.6 \pm 0.93$ & $101.4 \pm 0.75$ & $102.1 \pm 0.30$ \\
\hline Ceftriaxone sodium & $99.3 \pm 0.52$ & $100.6 \pm 0.96$ & $100.2 \pm 0.51$ \\
\hline Cefotaxime sodium & $99.7 \pm 1.35$ & $101.5 \pm 0.83$ & $101.3 \pm 1.16$ \\
\hline Cefixime & $98.3 \pm 1.24$ & $98.7 \pm 0.58$ & $98.6 \pm 0.73$ \\
\hline Cefazolin sodium & $101.1 \pm 1.08$ & $98.9 \pm 0.60$ & $102.3 \pm 0.68$ \\
\hline \multirow[t]{2}{*}{ Cefpodoxime proxetil } & $99.4 \pm 0.35$ & $99.4 \pm 0.47$ & $99.0 \pm 0.29$ \\
\hline & \multicolumn{3}{|c|}{ Recovery $(\%) \pm$ SD $^{a}$} \\
\hline Drug & $10 \mu \mathrm{g} \mathrm{mL}^{-1}$ & $15 \mu \mathrm{g} \mathrm{mL}^{-1}$ & $20 \mu \mathrm{g} \mathrm{mL}^{-1}$ \\
\hline Cefadroxil monohydrate & $99.9 \pm 1.31$ & $100.4 \pm 0.83$ & $99.1 \pm 0.90$ \\
\hline \multirow[t]{2}{*}{ Cefalexin anhydrous } & $98.6 \pm 0.26$ & $102.2 \pm 1.29$ & $98.0 \pm 0.41$ \\
\hline & \multicolumn{3}{|c|}{ Recovery $(\%) \pm \mathrm{SD}^{\mathrm{a}}$} \\
\hline Drug & $40 \mu \mathrm{g} \mathrm{mL} \mathrm{L}^{-1}$ & $80 \mu \mathrm{g} \mathrm{mL} L^{-1}$ & $120 \mu \mathrm{g} \mathrm{mL}^{-1}$ \\
\hline Ceftazidime pentahydrate & $102.3 \pm 0.86$ & $98.9 \pm 1.25$ & $99.6 \pm 0.82$ \\
\hline \multirow[t]{2}{*}{ Cefoperazone sodium } & $98.0 \pm 0.70$ & $100.3 \pm 1.11$ & $101.4 \pm 1.03$ \\
\hline & \multicolumn{3}{|c|}{ Recovery $(\%) \pm$ SD $^{a}$} \\
\hline Drug & $15 \mu \mathrm{g} \mathrm{mL^{-1 }}$ & $30 \mu \mathrm{g} \mathrm{mL}^{-1}$ & $45 \mu \mathrm{g} \mathrm{mL^{-1 }}$ \\
\hline Cefradine anhydrous & $98.3 \pm 0.51$ & $100.8 \pm 0.66$ & $100.7 \pm 0.87$ \\
\hline
\end{tabular}

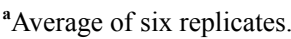

Table 6. Intra- and inter-day precision of the proposed spectrophotometric method.

\begin{tabular}{|c|c|c|c|c|c|}
\hline \multirow{2}{*}{ Drug } & \multirow{2}{*}{$\begin{array}{c}\text { Drug Conc. }(\mu \mathrm{g} \\
\left.\mathrm{mL}^{-1}\right)\end{array}$} & \multicolumn{2}{|c|}{ Intra-day precision } & \multicolumn{2}{|c|}{ Inter-day precision } \\
\hline & & $\operatorname{Mean} \pm \mathbf{S D}^{\mathbf{a}}$ & \% RSD & $\operatorname{Mean} \pm \mathbf{S D}^{\mathrm{a}}$ & $\%$ RSD \\
\hline \multirow[t]{3}{*}{ Cefaclor monohydrate } & 25 & $99.6 \pm 0.93$ & 0.93 & $99.4 \pm 1.29$ & 1.30 \\
\hline & 50 & $99.9 \pm 1.65$ & 1.66 & $98.5 \pm 0.90$ & 0.91 \\
\hline & 75 & $100.1 \pm 1.40$ & 1.40 & $100.7 \pm 1.12$ & 1.12 \\
\hline \multirow[t]{3}{*}{ Cefalexin anhydrous } & 10 & $100.3 \pm 1.17$ & 1.16 & $98.9 \pm 1.23$ & 1.25 \\
\hline & 15 & $100.4 \pm 1.53$ & 1.52 & $99.0 \pm 0.97$ & 0.98 \\
\hline & 20 & $100.1 \pm 1.16$ & 1.16 & $100.5 \pm 0.88$ & 0.88 \\
\hline \multirow[t]{3}{*}{ Cefadroxil monohydrate } & 10 & $99.4 \pm 0.99$ & 1.00 & $101.0 \pm 1.09$ & 1.08 \\
\hline & 15 & $99.9 \pm 0.85$ & 0.85 & $100.5 \pm 0.77$ & 0.76 \\
\hline & 20 & $100.2 \pm 1.37$ & 1.37 & $98.7 \pm 0.68$ & 0.69 \\
\hline \multirow[t]{3}{*}{ Cefradine anhydrous } & 15 & $100.1 \pm 1.03$ & 1.02 & $99.5 \pm 1.13$ & 1.14 \\
\hline & 30 & $100.0 \pm 1.15$ & 1.15 & $98.4 \pm 0.85$ & 0.86 \\
\hline & 45 & $100.6 \pm 1.16$ & 1.15 & $100.4 \pm 1.54$ & 1.54 \\
\hline \multirow[t]{3}{*}{ Cefoperazone sodium } & 40 & $99.6 \pm 0.93$ & 0.93 & $101.3 \pm 1.43$ & 1.41 \\
\hline & 80 & $99.7 \pm 0.67$ & 0.67 & $101.7 \pm 1.63$ & 1.61 \\
\hline & 120 & $100.3 \pm 1.35$ & 1.34 & $99.1 \pm 1.40$ & 1.41 \\
\hline \multirow[t]{3}{*}{ Ceftazidime pentahydrate } & 40 & $100.0 \pm 1.39$ & 1.39 & $100.9 \pm 0.99$ & 0.98 \\
\hline & 80 & $99.6 \pm 1.30$ & 1.31 & $101.3 \pm 1.27$ & 1.25 \\
\hline & 120 & $99.9 \pm 1.04$ & 1.05 & $98.9 \pm 1.12$ & 1.13 \\
\hline
\end{tabular}




\begin{tabular}{|c|c|c|c|c|c|}
\hline \multirow{2}{*}{ Drug } & \multirow{2}{*}{$\begin{array}{c}\text { Drug Conc. }(\mu \mathrm{g} \\
\left.\mathrm{mL}^{-1}\right)\end{array}$} & \multicolumn{2}{|c|}{ Intra-day precision } & \multicolumn{2}{|c|}{ Inter-day precision } \\
\hline & & Mean \pm SD $^{\mathbf{a}}$ & $\%$ RSD & $\operatorname{Mean} \pm \mathbf{S D}^{\mathbf{a}}$ & $\%$ RSD \\
\hline \multirow[t]{3}{*}{ Ceftriaxone sodium } & 25 & $100.4 \pm 1.57$ & 1.56 & $99.2 \pm 0.99$ & 1.00 \\
\hline & 50 & $101.0 \pm 1.27$ & 1.26 & $100.8 \pm 1.15$ & 1.14 \\
\hline & 75 & $99.8 \pm 1.38$ & 1.39 & $98.6 \pm 1.54$ & 1.57 \\
\hline \multirow[t]{3}{*}{ Cefotaxime sodium } & 25 & $99.3 \pm 1.05$ & 1.06 & $99.0 \pm 0.77$ & 0.78 \\
\hline & 50 & $98.8 \pm 0.78$ & 0.79 & $101.4 \pm 1.46$ & 1.44 \\
\hline & 75 & $99.4 \pm 1.29$ & 1.30 & $98.5 \pm 0.91$ & 0.93 \\
\hline \multirow[t]{3}{*}{ Cefixime } & 25 & $99.5 \pm 0.81$ & 0.81 & $100.9 \pm 0.99$ & 0.98 \\
\hline & 50 & $99.8 \pm 1.02$ & 1.03 & $99.7 \pm 1.17$ & 1.17 \\
\hline & 75 & $99.6 \pm 1.48$ & 1.48 & $98.3 \pm 1.65$ & 1.67 \\
\hline \multirow[t]{3}{*}{ Cefazolin sodium } & 25 & $100.5 \pm 1.15$ & 1.14 & $100.6 \pm 0.68$ & 0.67 \\
\hline & 50 & $100.6 \pm 1.36$ & 1.35 & $99.6 \pm 1.35$ & 1.35 \\
\hline & 75 & $101.4 \pm 0.74$ & 0.73 & $98.8 \pm 1.12$ & 1.14 \\
\hline \multirow[t]{3}{*}{ Cefpodoxime proxetil } & 25 & $100.4 \pm 0.89$ & 0.89 & $101.3 \pm 0.77$ & 0.76 \\
\hline & 50 & $100.5 \pm 1.15$ & 1.15 & $99.7 \pm 1.55$ & 1.55 \\
\hline & 75 & $100.0 \pm 1.70$ & 1.70 & $100.6 \pm 1.63$ & 1.62 \\
\hline
\end{tabular}

${ }^{\mathrm{a} A v e r a g e ~ o f ~ s i x ~ d e t e r m i n a t i o n s . ~}$

Table 7. Robustness of the proposed spectrophotometric method.

\begin{tabular}{|c|c|c|c|c|c|c|}
\hline \multirow[b]{2}{*}{ Experimental parameter variation } & \multicolumn{6}{|c|}{$\operatorname{Recovery}(\%) \pm \mathbf{S D}^{\mathbf{a}}$} \\
\hline & $\begin{array}{c}\text { Cefadroxil } \\
\text { monohydrate } \\
\left(20 \mu \mathrm{g} \mathrm{mL}^{-1}\right)\end{array}$ & $\begin{array}{c}\text { Cefalexin } \\
\text { anhydrous } \\
\left(20 \mu \mathrm{g} \mathrm{mL}^{-1}\right)\end{array}$ & $\begin{array}{c}\text { Cefradine } \\
\text { anhydrous } \\
\left(40 \mu \mathrm{g} \mathrm{mL}^{-1}\right)\end{array}$ & $\begin{array}{c}\text { Cefaclor } \\
\text { monohydrate } \\
\left(60 \mu \mathrm{g} \mathrm{mL}^{-1}\right)\end{array}$ & $\begin{array}{c}\text { Cefopera- } \\
\text { zone sodium } \\
\left(80 \mu \mathrm{g} \mathrm{mL}^{-1}\right)\end{array}$ & $\begin{array}{l}\text { Ceftazidime } \\
\text { pentahydrate } \\
\left(80 \mu \mathrm{g} \mathrm{mL}^{-1}\right)\end{array}$ \\
\hline No variation ${ }^{b}$ & $99.4 \pm 1.21$ & $99.8 \pm 0.31$ & $99.5 \pm 1.82$ & $99.5 \pm 1.13$ & $99.2 \pm 0.56$ & $99.5 \pm 0.47$ \\
\hline $\begin{array}{c}\text { 1- } \mathrm{NaOH} \text { concentration } \\
0.45 \mathrm{M} \\
0.55 \mathrm{M}\end{array}$ & $\begin{array}{l}98.3 \pm 0.85 \\
98.6 \pm 1.19\end{array}$ & $\begin{array}{c}97.9 \pm 1.20 \\
100.9 \pm 1.15\end{array}$ & $\begin{array}{l}101.5 \pm 1.32 \\
98.2 \pm 0.52\end{array}$ & $\begin{array}{r}97.5 \pm 0.54 \\
101.3 \pm 0.77\end{array}$ & $\begin{array}{c}101.8 \pm 1.11 \\
99.4 \pm 1.31\end{array}$ & $\begin{array}{l}97.4 \pm 1.12 \\
98.3 \pm 1.34\end{array}$ \\
\hline $\begin{array}{l}\text { 2- NBD-Cl concentration } \\
\begin{array}{c}2.8 \times 10^{-3} \mathrm{M} \\
3.2 \times 10^{-3} \mathrm{M}\end{array}\end{array}$ & $\begin{array}{c}102.0 \pm 0.25 \\
98.5 \pm 1.31\end{array}$ & $\begin{array}{c}100.3 \pm 1.35 \\
100.9 \pm 0.92\end{array}$ & $\begin{array}{l}98.2 \pm 1.56 \\
98.0 \pm 1.15\end{array}$ & $\begin{array}{r}102.1 \pm 0.35 \\
98.5 \pm 0.91\end{array}$ & $\begin{array}{c}100.9 \pm 1.58 \\
98.5 \pm 0.83\end{array}$ & $\begin{array}{l}100.9 \pm 1.15 \\
102.0 \pm 0.88\end{array}$ \\
\hline $\begin{array}{l}\text { 3- Heating temperature } \\
95^{\circ} \mathrm{C} \\
100^{\circ} \mathrm{C}\end{array}$ & $\begin{array}{l}98.8 \pm 0.78 \\
99.6 \pm 1.35\end{array}$ & $\begin{array}{c}97.9 \pm 1.20 \\
100.8 \pm 1.60\end{array}$ & $\begin{array}{c}102.4 \pm 156 \\
100.4 \pm 0.90\end{array}$ & $\begin{array}{l}102.7 \pm 2.21 \\
99.8 \pm 1.02\end{array}$ & $\begin{array}{l}101.0 \pm 1.27 \\
101.3 \pm 1.27\end{array}$ & $\begin{array}{c}101.4 \pm 2.04 \\
98.0 \pm 2.15\end{array}$ \\
\hline $\begin{array}{l}\text { 4- Heating time } \\
25 \mathrm{~min} \\
35 \mathrm{~min} \\
\end{array}$ & $\begin{array}{l}100.5 \pm 1.23 \\
100.1 \pm 1.40\end{array}$ & $\begin{array}{l}99.2 \pm 0.99 \\
100.4 \pm 1.53\end{array}$ & $\begin{array}{l}98.5 \pm 1.57 \\
98.7 \pm 0.68\end{array}$ & $\begin{array}{l}97.5 \pm 1.98 \\
99.6 \pm 0.93\end{array}$ & $\begin{array}{r}99.1 \pm 1.40 \\
100.9 \pm 0.99\end{array}$ & $\begin{array}{c}99.4 \pm 1.29 \\
98.5 \pm 0.90\end{array}$ \\
\hline \multicolumn{7}{|l|}{ Table 7. (Continued). } \\
\hline & \multicolumn{6}{|c|}{ Recovery $(\%) \pm$ SD $^{\mathbf{a}}$} \\
\hline Experimental parameter variation & $\begin{array}{c}\text { Ceftriaxone so- } \\
\text { dium } \\
\left(60 \mu \mathrm{g} \mathrm{mL}^{-1}\right)\end{array}$ & $\begin{array}{r}\text { Cefota } \\
\text { sodiı } \\
(60 \mu \mathrm{g} \\
\end{array}$ & $\begin{array}{l}\text { me } \\
\left.L^{-1}\right)\end{array}$ & $\begin{array}{l}\text { ixime } \\
\left.\text { g mL }^{-1}\right)\end{array}$ & $\begin{array}{c}\text { Cefazolin } \\
\text { sodium } \\
\left(60 \mu \mathrm{g} \mathrm{mL}^{-1}\right) \\
\end{array}$ & $\begin{array}{l}\text { Cefpodoxime } \\
\text { proxetil } \\
\left(60 \mu \mathrm{g} \mathrm{mL}^{-1}\right) \\
\end{array}$ \\
\hline No variation $^{\mathbf{b}}$ & $99.5 \pm 0.66$ & $97.6 \pm$ & 100 & $7 \pm 0.98$ & $99.5 \pm 1.23$ & $99.5 \pm 1.01$ \\
\hline $\begin{array}{c}\text { 1- } \mathrm{NaOH} \text { concentration } \\
0.45 \mathrm{M} \\
0.55 \mathrm{M}\end{array}$ & $\begin{array}{l}100.2 \pm 1.35 \\
98.7 \pm 0.98\end{array}$ & $\begin{array}{c}99.5 \pm \\
98.3 \pm\end{array}$ & $\begin{array}{l}99 . \\
98 .\end{array}$ & $\begin{array}{l} \pm 1.27 \\
\pm 0.73\end{array}$ & $\begin{array}{r}98.6 \pm 0.88 \\
102.4 \pm 1.145\end{array}$ & $\begin{array}{c}98.1 \pm 0.60 \\
99.5 \pm 1.27\end{array}$ \\
\hline $\begin{array}{l}\text { 2- NBD-Cl concentration } \\
\begin{array}{l}2.8 \times 10^{-3} \mathrm{M} \\
3.2 \times 10^{-3} \mathrm{M}\end{array}\end{array}$ & $\begin{array}{c}99.6 \pm 1.15 \\
98.0 \pm 0.75\end{array}$ & $\begin{array}{c}102.0 \pm \\
102.3 \pm\end{array}$ & $\begin{array}{l}102 \\
99.5\end{array}$ & $\begin{array}{l}3 \pm 1.30 \\
\pm 0.69\end{array}$ & $\begin{array}{r}99.7 \pm 1.35 \\
101.5 \pm 0.70\end{array}$ & $\begin{array}{c}98.4 \pm 1.40 \\
99.9 \pm 0.49\end{array}$ \\
\hline $\begin{array}{c}\text { 3- Heating temperature } \\
95^{\circ} \mathrm{C} \\
100^{\circ} \mathrm{C}\end{array}$ & $\begin{array}{l}99.0 \pm 1.30 \\
97.5 \pm 0.68\end{array}$ & $\begin{array}{r}99.4 \pm \\
98.4 \pm 1\end{array}$ & $\begin{array}{r}98 . \\
101.9\end{array}$ & $\begin{array}{l} \pm 0.66 \\
\pm 1.35\end{array}$ & $\begin{array}{l}100.6 \pm 0.48 \\
97.5 \pm 1.44\end{array}$ & $\begin{array}{l}100.8 \pm 0.72 \\
99.1 \pm 0.65\end{array}$ \\
\hline $\begin{array}{l}\text { 4- Heating time } \\
25 \mathrm{~min} \\
35 \mathrm{~min}\end{array}$ & $\begin{array}{c}101.1 \pm 1.44 \\
98.1 \pm 0.81\end{array}$ & $\begin{array}{c}97.8 \pm \\
101.4 \pm\end{array}$ & $\begin{array}{l}90 \\
.41\end{array}$ & $\begin{array}{l} \pm 0.56 \\
\pm 1.27\end{array}$ & $\begin{array}{c}99.7 \pm 0.70 \\
101.9 \pm 0.72\end{array}$ & $\begin{array}{l}97.8 \pm 0.49 \\
98.0 \pm 1.34\end{array}$ \\
\hline
\end{tabular}

average of three determinations;

${ }^{\mathbf{b}}$ Following the general assay procedure conditions. 
Table 8. Determination of the studied drugs in their pharmaceutical dosage forms.

\begin{tabular}{|c|c|c|c|}
\hline \multirow[b]{2}{*}{ Drug } & \multirow[b]{2}{*}{ Pharmaceutical product } & \multicolumn{2}{|c|}{ Recovery $\% \pm$ SD } \\
\hline & & $\begin{array}{l}\text { Proposed method } \\
(n=6)\end{array}$ & $\begin{array}{c}\begin{array}{c}\text { Reported method } \\
(n=6)\end{array} \\
\end{array}$ \\
\hline \multirow[t]{2}{*}{$\begin{array}{l}\text { Cefaclor } \\
\text { monohydrate }\end{array}$} & $\begin{array}{l}\text { Ceclor }^{\circledR} \text { suspension }^{\mathrm{c}} \\
250 \mathrm{mg} \text { of cefaclor monohydrate } / 5 \mathrm{~mL}\end{array}$ & $\begin{array}{c}97.8 \pm 0.5, \mathrm{t}=0.382^{\mathrm{a}} \\
\mathrm{F}=1.562^{\mathrm{a}}\end{array}$ & $97.7 \pm 0.40$ \\
\hline & $\begin{array}{l}\text { Bacticlor }^{\mathbb{Q}} \text { suspension }^{\mathrm{d}} \\
250 \mathrm{mg} \text { of cefaclor anhydrous } / 5 \mathrm{~mL}\end{array}$ & $\begin{array}{c}97.2 \pm 0.5, \mathrm{t}=1.913 \\
\mathrm{~F}=1.562\end{array}$ & $96.7 \pm 0.40$ \\
\hline \multirow[t]{5}{*}{$\begin{array}{l}\text { Cefadroxil } \\
\text { monohydrate }\end{array}$} & $\begin{array}{l}\text { Duricef }^{\mathbb{B}} \text { tablets }^{\mathrm{e}} \\
1 \mathrm{~g} \text { of cefadroxil monohydrate/tablet }\end{array}$ & $\begin{array}{c}98.7 \pm 0.3, \mathrm{t}=2.038 \\
\mathrm{~F}=2.250\end{array}$ & $99.0 \pm 0.20$ \\
\hline & $\begin{array}{l}\text { Duricef }^{(\mathbb{B}} \text { suspension }^{\mathrm{e}} \\
250 \mathrm{mg} \text { of cefadroxil monohydrate } / 5 \mathrm{~mL}\end{array}$ & $\begin{array}{c}96.6 \pm 1.3, \mathrm{t}=1.605 \\
\mathrm{~F}=2.641\end{array}$ & $97.6 \pm 0.80$ \\
\hline & $\begin{array}{l}\text { Duricef }^{\mathrm{B}} \text { capsules }^{\mathrm{e}} \\
500 \mathrm{mg} \text { of cefadroxil monohydrate/capsule }\end{array}$ & $\begin{array}{c}97.9 \pm 1.3, \mathrm{t}=1.332 \\
\mathrm{~F}=1.032\end{array}$ & $98.9 \pm 1.30$ \\
\hline & $\begin{array}{l}\text { Biodroxil }^{\circledR} \text { capsules }^{\mathrm{f}} \\
500 \mathrm{mg} \text { of cefadroxil monohydrate/capsule }\end{array}$ & $\begin{array}{c}102.4 \pm 1.4, \mathrm{t}=0.930 \\
\mathrm{~F}=1.361\end{array}$ & $101.7 \pm 1.20$ \\
\hline & $\begin{array}{l}\text { Biodroxil }^{\circledR} \text { suspension }^{\mathrm{f}} \\
250 \mathrm{mg} \text { of cefadroxil monohydrate } / 5 \mathrm{~mL}\end{array}$ & $\begin{array}{c}103.1 \pm 0.6, \mathrm{t}=1.359 \\
\mathrm{~F}=2.250\end{array}$ & $102.7 \pm 0.40$ \\
\hline \multirow[t]{3}{*}{$\begin{array}{l}\text { Cefalexin } \\
\text { anhydrous }\end{array}$} & $\begin{array}{l}\text { Ceporex }^{\circledR} \text { tablets }^{\mathrm{g}} \\
500 \mathrm{mg} \text { of cefalexin anhydrous/tablet }\end{array}$ & $\begin{array}{c}99.3 \pm 1.6, \mathrm{t}=0.646 \\
\mathrm{~F}=1.778\end{array}$ & $98.7 \pm 1.20$ \\
\hline & $\begin{array}{l}\text { Ceporex }^{\circledR} \text { suspension }{ }^{\mathrm{g}} \\
250 \mathrm{mg} \text { of cefalexin anhydrous } / 5 \mathrm{~mL}\end{array}$ & $\begin{array}{c}99.0 \pm 1.5, \mathrm{t}=0.735 \\
\mathrm{~F}=3.516\end{array}$ & $98.5 \pm 0.80$ \\
\hline & $\begin{array}{l}\text { Ospexin }^{\circledR} \text { suspension }^{\mathrm{h}} \\
250 \mathrm{mg} \text { of cefalexin anhydrous } / 5 \mathrm{~mL}\end{array}$ & $\begin{array}{c}103.5 \pm 1.5, \mathrm{t}=0.576 \\
\mathrm{~F}=3.516\end{array}$ & $103.1 \pm 0.80$ \\
\hline \multirow[t]{4}{*}{$\begin{array}{l}\text { Cefradine } \\
\text { anhydrous }\end{array}$} & $\begin{array}{l}\text { Velosef }^{\circledR} \text { capsules }^{\mathrm{e}} \\
250 \mathrm{mg} \text { of cefradine anhydrous/capsule }\end{array}$ & $\begin{array}{c}97.7 \pm 0.5, \mathrm{t}=1.530 \\
\mathrm{~F}=1.562\end{array}$ & $97.3 \pm 0.40$ \\
\hline & $\begin{array}{l}\text { Velosef }^{\circledR} \text { tablets } \\
1 \mathrm{~g} \text { of cefradine anhydrous/tablet }\end{array}$ & $\begin{array}{c}103.3 \pm 1.2, \mathrm{t}=1.019 \\
\mathrm{~F}=2.250\end{array}$ & $102.7 \pm 0.80$ \\
\hline & $\begin{array}{l}\text { Velosef }^{\mathbb{B}} \text { suspension }^{\mathrm{e}} \\
205 \mathrm{mg} \text { of cefradine anhydrous } / 5 \mathrm{~mL}\end{array}$ & $\begin{array}{c}99.0 \pm 1.5, \mathrm{t}=0.588 \\
\mathrm{~F}=3.516\end{array}$ & $98.5 \pm 0.80$ \\
\hline & $\begin{array}{l}\text { Velosef }^{\mathbb{B}} \text { vials }^{\mathrm{e}} \\
1 \mathrm{~g} \text { of cefradine anhydrous/vial }\end{array}$ & $\begin{array}{c}97.9 \pm 1.2, \mathrm{t}=0.267 \\
\mathrm{~F}=1.778\end{array}$ & $97.7 \pm 0.90$ \\
\hline $\begin{array}{l}\text { Cefotaxime } \\
\text { sodium }\end{array}$ & 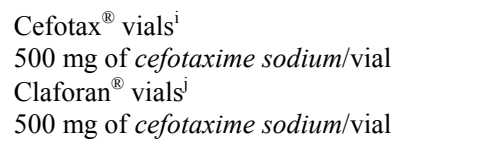 & $\begin{array}{l}98.2 \pm 1.8, \mathrm{t}=0.365 \\
\mathrm{~F}=3.932 \\
97.9 \pm 1.2, \mathrm{t}=0.326 \\
\mathrm{~F}=1.778\end{array}$ & $\begin{array}{l}97.9 \pm 0.90 \\
97.7 \pm 0.90\end{array}$ \\
\hline $\begin{array}{l}\text { Ceftazidime } \\
\text { pentahydrate }\end{array}$ & $\begin{array}{l}\text { Fortum }^{\circledR} \text { vials }^{\mathrm{k}} \\
500 \mathrm{mg} \text { of ceftazidime pentahydrate/vial }\end{array}$ & $\begin{array}{l}98.9 \pm 0.6, \mathrm{t}=1.460 \\
\mathrm{~F}=4.001\end{array}$ & $98.5 \pm 0.30$ \\
\hline $\begin{array}{l}\text { Cefoperazone } \\
\text { sodium }\end{array}$ & $\begin{array}{l}\text { Cefozon }^{\circledR} \text { vials }^{1} \\
500 \mathrm{mg} \text { of cefoperazone sodium/vial }\end{array}$ & $\begin{array}{c}102.3 \pm 1.4, \mathrm{t}=1.721 \\
\mathrm{~F}=4.003\end{array}$ & $101.2 \pm 0.70$ \\
\hline $\begin{array}{l}\text { Ceftriaxone } \\
\text { sodium }\end{array}$ & $\begin{array}{l}\text { Ceftriaxone }^{\circledR} \text { vials }^{\mathrm{m}} \\
500 \mathrm{mg} \text { of } \text { ceftriaxone sodium/vial }^{2}\end{array}$ & $\begin{array}{c}97.7 \pm 0.4, \mathrm{t}=1.643 \\
\mathrm{~F}=4.002\end{array}$ & $98.0 \pm 0.20$ \\
\hline Cefixime & $\begin{array}{l}\text { Ximacef }^{\circledR} \text { capsules }^{\mathrm{n}} \\
400 \mathrm{mg} \text { of cefixime/capsule }\end{array}$ & $\begin{array}{c}102.1 \pm 1.4, \mathrm{t}=0.797 \\
\mathrm{~F}=1.361\end{array}$ & $101.5 \pm 1.20$ \\
\hline $\begin{array}{l}\text { Cefazolin } \\
\text { sodium }\end{array}$ & $\begin{array}{l}\text { Zinol }^{\circledR} \text { vials }^{0} \\
500 \mathrm{mg} \text { of } \text { cefazolin sodium/vial }\end{array}$ & $\begin{array}{c}98.9 \pm 1.3, \mathrm{t}=1.493 \\
\mathrm{~F}=3.449\end{array}$ & $98.0 \pm 0.70$ \\
\hline $\begin{array}{l}\text { Cefpodoxime } \\
\text { proxetil }\end{array}$ & $\begin{array}{l}\text { Orelox }^{\circledR} \text { tablets }^{q} \\
100 \mathrm{mg} \text { of cefpodoxime proxetil/tablet }\end{array}$ & $\begin{array}{c}98.2 \pm 1.8, \mathrm{t}=0.298 \\
\mathrm{~F}=3.932\end{array}$ & $97.9 \pm 0.90$ \\
\hline
\end{tabular}

${ }^{\mathrm{a}}$ Theoretical value for $\mathrm{t}$ and $\mathrm{F}$ at $95 \%$ confidence limit, $\mathrm{t}=2.228$ and $\mathrm{F}=5.053 ;{ }^{\mathrm{b}}$ Reference 48 ; ${ }^{\mathrm{c}}$ Egyptian Pharmaceuticals and chemicals industries Co., S.A.E., Bayad El-Arab, Beni Suef, Egypt; ${ }^{\mathbf{d}}$ Pharco Pharmaceuticals, Alexandria under license from Ranbaxy UK; ${ }^{\mathrm{e}}$ Bristol-Myers Squibb Pharmaceutical Co., Cairo, Egypt; ${ }^{\mathrm{f}}$ Kahira Pharm. \& Chem. Ind. Co. under license from Novartis Pharma S.A.E., Cairo, Egypt; 
${ }^{\mathrm{g}}$ GlaxoSmithKline, S.A.E., El Salam City, Cairo, Egypt; ${ }^{\text {h }}$ Pharco Pharmaceuticals, Alexandria under license from Biochemie GmbH., Vienna, Austria; ${ }^{\mathrm{i}}$ T3A Pharma Group, Assiut, Egypt $;{ }^{j}$ Hoechst Orient, S.A.E., Cairo, Egypt ; ${ }^{\mathrm{k}}$ GalaxoWellcome, S.A.E., El Salam City, Cairo, Egypt; ${ }^{\mathrm{l} E g y p t i a n}$ International Pharmaceutical Industries Co., El Asher Ramadan City, Cairo, Egypt; ${ }^{\mathrm{m}}$ Kahira Pharm. \& Chem. Ind. Co. under licence from Novartis Pharma S.A.E., Cairo, Egypt; " Sigma pharmaceutical industries, S.A.E., Egypt; ${ }^{\circ}$ Pharco Pharmaceuticals, Alexandria, Egypt; ${ }^{\mathrm{q}}$ Aventis, Zeitoun, Cairo, Egypt.

Table 9. Standard addition method for the assay of the studied drugs in their pharmaceutical dosage forms by the proposed method.

\begin{tabular}{|c|c|c|c|c|}
\hline Drug & $\begin{array}{l}\text { Pharmaceutical } \\
\text { formulation }\end{array}$ & $\begin{array}{c}\text { Authentic drug added } \\
\left(\mu \mathrm{g} \mathrm{mL} \mathrm{L}^{-1}\right)\end{array}$ & $\begin{array}{l}\text { Authentic drug found } \\
\left(\mu \mathrm{g} \mathrm{mL} \mathrm{L}^{-1}\right)\end{array}$ & Recovery $(\%) \pm S^{a}$ \\
\hline \multirow{6}{*}{$\begin{array}{l}\text { Cefaclor } \\
\text { monohydrate }\end{array}$} & \multirow{3}{*}{ Ceclor $^{\circledR}$ suspension } & 10.00 & 9.97 & $99.7 \pm 1.21$ \\
\hline & & 20.00 & 19.40 & $97.0 \pm 1.80$ \\
\hline & & 30.00 & 28.83 & $96.1 \pm 1.52$ \\
\hline & \multirow{3}{*}{ Bacticlor $^{\circledR}$ suspension } & 10.00 & 10.06 & $100.6 \pm 1.10$ \\
\hline & & 20.00 & 19.26 & $96.3 \pm 0.81$ \\
\hline & & 30.00 & 29.40 & $98.0 \pm 0.90$ \\
\hline \multirow{15}{*}{$\begin{array}{l}\text { Cefadroxil } \\
\text { monohydrate }\end{array}$} & \multirow{3}{*}{$\begin{array}{l}\text { Duricef }^{(\mathbb{R}} \\
\text { tablets }\end{array}$} & 5.00 & 5.11 & $102.2 \pm 1.41$ \\
\hline & & 10.00 & 9.95 & $99.5 \pm 1.72$ \\
\hline & & 15.00 & 14.85 & $99.0 \pm 0.50$ \\
\hline & \multirow{3}{*}{ Duricef $^{\circledR}$ suspension } & 5.00 & 4.90 & $98.0 \pm 1.81$ \\
\hline & & 10.00 & 10.02 & $100.2 \pm 1.63$ \\
\hline & & 15.00 & 14.64 & $97.6 \pm 1.12$ \\
\hline & \multirow{3}{*}{ Duricef $^{\circledR}$ capsules } & 5.00 & 4.83 & $96.6 \pm 1.50$ \\
\hline & & 10.00 & 9.60 & $96.0 \pm 1.30$ \\
\hline & & 15.00 & 14.78 & $98.5 \pm 0.71$ \\
\hline & \multirow{4}{*}{ Biodroxil $^{\circledR}$ capsules } & 5.00 & 4.89 & $97.8 \pm 1.54$ \\
\hline & & 10.00 & 10.36 & $103.6 \pm 0.91$ \\
\hline & & 15.00 & 14.98 & $99.9 \pm 0.60$ \\
\hline & & 5.00 & 4.87 & $97.4 \pm 1.91$ \\
\hline & \multirow[t]{3}{*}{ Biodroxil $^{\circledR}$ suspension } & 10.00 & 9.84 & $98.4 \pm 0.42$ \\
\hline & & 15.00 & 15.38 & $102.5 \pm 1.20$ \\
\hline & & 5.00 & 4.98 & $99.6 \pm 1.41$ \\
\hline \multirow{8}{*}{ Cefalexin anhydrous } & \multirow[t]{2}{*}{ Ceporex $^{\circledR}$ tablets } & 10.00 & 10.07 & $100.7 \pm 1.73$ \\
\hline & & 15.00 & 14.82 & $98.8 \pm 0.62$ \\
\hline & \multirow{4}{*}{ Ceporex ${ }^{\circledR}$ suspension } & 5.00 & 5.05 & $101.0 \pm 1.00$ \\
\hline & & 10.00 & 9.79 & $97.9 \pm 1.81$ \\
\hline & & 15.00 & 15.06 & $100.4 \pm 0.91$ \\
\hline & & 5.00 & 4.95 & $99.0 \pm 1.30$ \\
\hline & \multirow{3}{*}{ Ospexin $^{\circledR}$ suspension } & 10.00 & 9.92 & $99.2 \pm 1.81$ \\
\hline & & 15.00 & 14.68 & $97.9 \pm 1.94$ \\
\hline \multirow{12}{*}{$\begin{array}{l}\text { Cefradine } \\
\text { anhydrous }\end{array}$} & & 10.00 & 9.85 & $98.5 \pm 0.85$ \\
\hline & \multirow[t]{2}{*}{ Velosef $^{\circledR}$ capsules $^{-}$} & 20.00 & 20.09 & $100.5 \pm 0.63$ \\
\hline & & 30.00 & 28.86 & $96.2 \pm 1.11$ \\
\hline & \multirow{3}{*}{$\begin{array}{l}\text { Velosef }^{\mathbb{B}} \\
\text { tablets }\end{array}$} & 10.00 & 9.69 & $96.9 \pm 0.80$ \\
\hline & & 20.00 & 19.85 & $99.3 \pm 0.50$ \\
\hline & & 30.00 & 30.10 & $100.3 \pm 0.71$ \\
\hline & \multirow{3}{*}{ Velosef $^{\circledR}$ suspension } & 10.00 & 9.72 & $97.2 \pm 0.92$ \\
\hline & & 20.00 & 19.94 & $99.7 \pm 1.50$ \\
\hline & & 30.00 & 31.14 & $103.8 \pm 1.93$ \\
\hline & \multirow{3}{*}{$\begin{array}{l}\text { Velosef }^{\circledR} \\
\text { vials }\end{array}$} & 10.00 & 10.16 & $101.6 \pm 1.10$ \\
\hline & & 20.00 & 19.62 & $98.1 \pm 1.63$ \\
\hline & & 30.00 & 29.85 & $99.5 \pm 1.94$ \\
\hline & & 20.00 & 20.34 & $101.7 \pm 1.33$ \\
\hline Ceftazidime & Fortum & 40.00 & 38.76 & $96.6 \pm 1.63$ \\
\hline & & 60.00 & 61.32 & $102.2 \pm 1.32$ \\
\hline & Cefotax ${ }^{\circledR}$ & 10.00 & 9.66 & $96.6 \pm 0.7$ \\
\hline & Cetotax & 20.00 & 20.20 & $101.0 \pm 0.9$ \\
\hline Cefotaxime & Vlals & 30.00 & 29.30 & $97.6 \pm 1.5$ \\
\hline Sodium & Claforan ${ }^{\circledR}$ & 10.00 & 10.35 & $103.5 \pm 1.8$ \\
\hline & Clatoran & 20.00 & 19.60 & $98.0 \pm 1.6$ \\
\hline & & 30.00 & 29.19 & $97.3 \pm 0.7$ \\
\hline & & 20.00 & 19.50 & $97.5 \pm 1.8$ \\
\hline Cefoperazone & Cefozon & 40.00 & 38.88 & $97.2 \pm 0.9$ \\
\hline Sodium & vials & 60.00 & 59.46 & $99.1 \pm 0.7$ \\
\hline & & 10.00 & 10.08 & $100.8 \pm 1.4$ \\
\hline Ceftriaxone & Ceftriaxone vials & 20.00 & 19.36 & $96.8 \pm 1.5$ \\
\hline Sodium & & 30.00 & 30.98 & $103.3 \pm 1.3$ \\
\hline
\end{tabular}




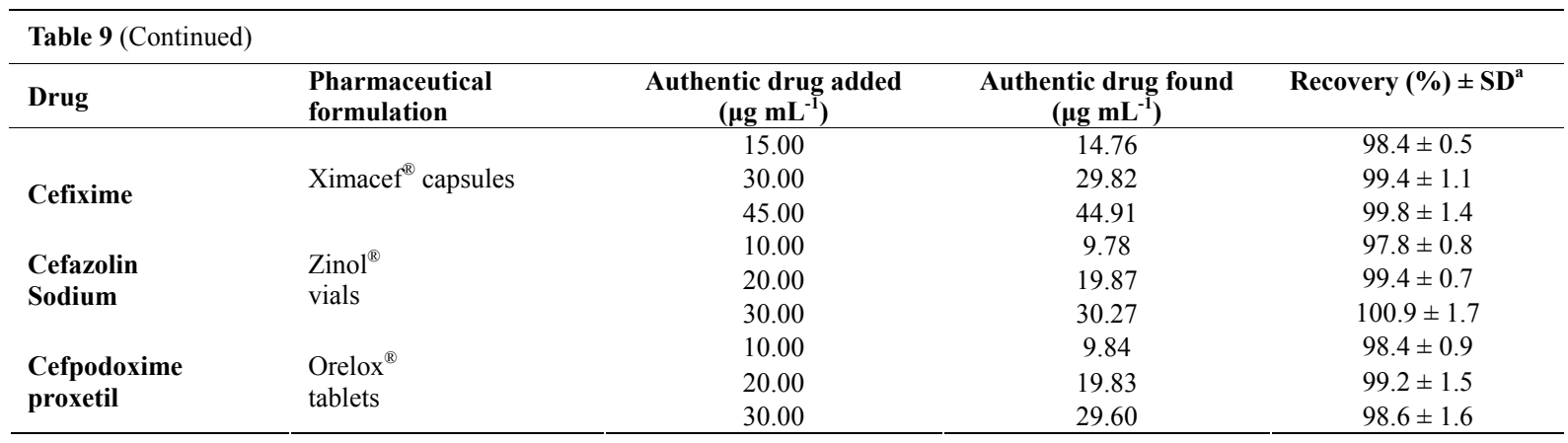

${ }^{\text {a }}$ Average of six determinations.

a good leaving group at position 4 in addition to the presence of nitro group as an electron withdrawing group at position 7 of the aromatic ring in NBD-Cl result in replacement of $\mathrm{Cl}^{-}$anion with the attacking sulfide ions which in turn lead to the formation of a yellowcolored chromophore $\left(\lambda_{\max }\right.$ at $\left.390 \mathrm{~nm}\right)$. The reaction product is stable in strong acidic medium, moreover acidification could minimize possible competition between the generated sulfide nucloephile and excess $\mathrm{OH}^{-}$ which may lead to decrease in the chromogen formed. The proposed reaction mechanism is given in the following scheme:

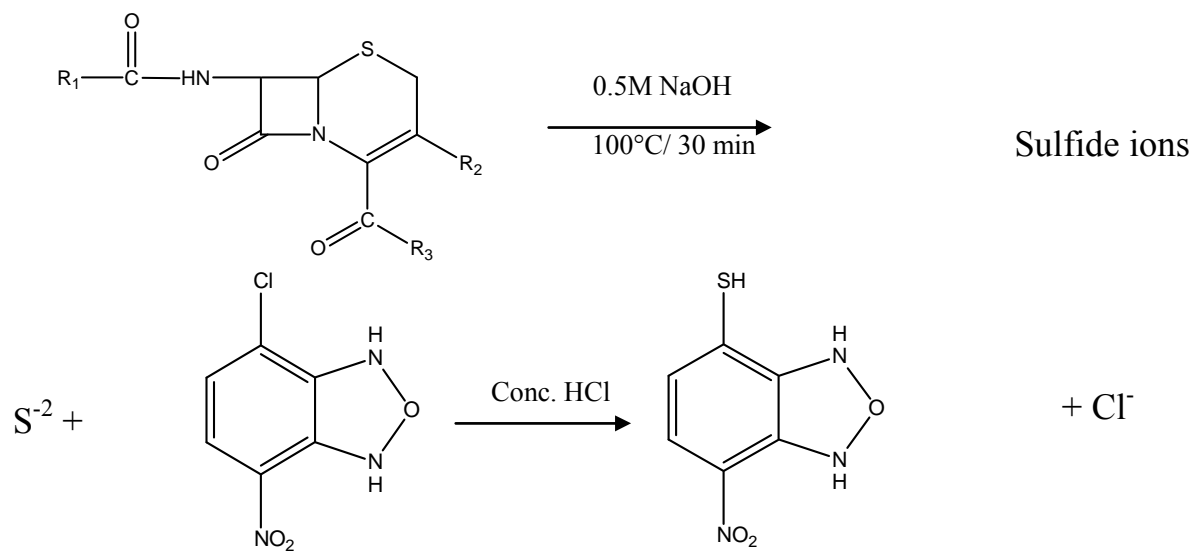

Scheme 1 Suggested reaction mechanism between sulfide ions and NBD-Cl

The production of sulfide ions was confirmed by carrying out specific qualitative tests such as dilute hydrochloric acid, cadmium acetate, sodium nitroprusside and methyelene blue tests [56] or by comparing $\lambda_{\max }$ of the formed chromogen with that obtained after applying the developed method to sodium sulfide and the same results were obtained.

\section{Conclusions}

The developed spectrophotometric method is precise, accurate and sensitive. No interference from the frequently encountered excipients and additives. Statistical analysis proves that the method could be applied for the analysis of the studied drugs in their pure forms and in pharmaceutical formulations.

\section{REFERENCES}

[1] Delgado, J.N. and Remers, W.A. (2004) Wilson and Gisvold's textbook of organic medicinal and pharmaceutical chemistry. 10th Edition, Lippincott Williams \& Wilkins, New York.

[2] Dollery, C. (1999) Therapeutic drugs. Vol. I. 3rd Edition, Churchill Livingstone, Edinburgh.

[3] American Pharmaceutical Association. (2008) United States Pharmacopoeia 31 and NF 26. Washington, DC.

[4] Abdel-Hamid, M.E. (1998) FSQ spectrophotometric and HPLC analysis of some cephalosporins in the presence of their alkali-induced degradation products. Il Farmaco, 53(2), 132-138.

[5] Kelani, K., Bebawy, L.I. and Abdel-Fattah, L. (1998) Stability-indicating spectrophotometric and densitometric methods for determination of some cephalosporins. Journal of AOAC International, 81(2), 386-393.

[6] El-Walily, A.F.M., Gazy, A.A., Belal, S.F. and Khamis, 
E.F. (2000) Quantitative determination of some thiazole cephalosporins through complexation with palladium (II) chloride. Journal of Pharmaceutical and Biomedical Analysis, 22(2), 385-392.

[7] Agbaba, D., Eric, S., Karljikovic-Rajic, K., Vladimirov, S. and Zivanov-Stakic, D. (1997) Spectrophotometric determination of certain cephalosporins using ferrihydroxamate method. Spectroscopy Letters, 30(2), 309-319.

[8] El-Walily, A.F.M., Gazy, A.A., Belal, S.F. and Khamis, E.F. (2000) Use of cerium (IV) in the spectrophotometric and spectrofluorimetric determinations of penicillins and cephalosporins in their pharmaceutical preparations. Spectroscopy Letters, 33(6), 931-948.

[9] Yang, J., Zhou, G.J., Cao, X.H., Ma, Q.L. and Dong, J. (1998) Study on the fluorescence characteristics of alkaline degradation of cefadroxil, cefradine, cefotaxime sodium and amoxicillin. Analytical Letters, 31(6), 10471060.

[10] Aly, F.A., Hefnawy, M.M. and Belal, F. (1996) A selective spectrofluorimetric method for the determination of some $\alpha$-aminocephalosporins in formulations and biological fluids. Analytical Letters, 29(1), 117-130.

[11] Yang, J.H, Ma, Q.L., Wu, X., Sun, L.M. and Cao, X.H. (1999) A new luminescence spectrometry for the determination of some $\beta$-lactamic antibiotics. Analytical Letters, 32(3), 471-480.

[12] Hefnawy, M., El-Shabrawy, Y. and Belal, F. (1999) Spectrofluorometric determination of alpha-aminocephalosporins in biological fluids and pharmaceutical preparations. Journal of Pharmaceutical and Biomedical Analysis, 21(4), 703-707.

[13] El-Walily, A.F.M., Gazy, A.A., Belal, S.F. and Khamis, E.F. (1999) Selective spectrofluorimetric determination of phenolic $\beta$-lactam antibiotics through the formation of their coumarin derivatives. Journal of Pharmaceutical and Biomedical Analysis, 20(4), 643-653.

[14] Li, Y. and Lu, J. (2006) Chemiluminescence flowinjection analysis of $\beta$-lactam antibiotics using the luminol-permanganate reaction. Luminescence, 21(4), 251255.

[15] Yao, H., Tang, Y., Li, Y. and Sun, Y. (2003) Flow injection chemiluminescence determination of cephalosporin antibiotics by their enhancing effects on luminal-potassium periodate system. Analytical Letters, 36(14), 2975-2983.

[16] Thongpoon, C., Liawruangrath, B., Liawruangrath, S., Wheatley, R.A. and Townshend, A. (2006) Flow injection chemiluminescence determination of cefadroxil using potassium permanganate and formaldehyde system. Journal of Pharmaceutical and Biomedical Analysis, 42(2), 277 282.

[17] Shinde, V.M. and Shabadi, C.V. (1997) Simultaneous determination of cefadroxil and cefalexin from capsules by reversed phase HPLC. Indian Drugs, 34, 399-402.

[18] Misztal, G. (1998) Determination of cefotaxime and cefotriaxone in pharmaceuticals by HPLC. Pharmazie, 53, 723-724.

[19] Shinde, V.M. and Shabadi, C.V. (1998) Simultaneous determination of ceazolin and cefotaxime from injections by reversed phase HPLC. Indian Journal of Pharmaceutical Sciences, 60(5), 313-315.

[20] LaCourse, W.R. and Dasenbrock C.O. (1999) Pulsed electrochemical detection of sulfur-containing antibiotics following high performance liquid chromatography. Journal of Pharmaceutical and Biomedical Analysis, 19(1-2), 239-252.

[21] Özkan, S.A., Erk, N., Uslu, B., Yilmaz, N. and Biryol, I. (2000) Study on electrooxidation of cefadroxil monohydrate and its determination by differential pulse voltammetry. Journal of Pharmaceutical and Biomedical Analysis, 23(2-3), 263-273.

[22] Rodrigues, L.N.C., Zanoni, M.B.V. and Fogg, A.G. (1999) Indirect polarographic and cathodic-stripping voltammetric determination of cefaclor as an alkaline degradation product. Journal of Pharmaceutical and Biomedical Analysis, 21(3), 497-505.

[23] El-Maali, N.A., Ali, A.M.M. and Ghandour, M.A. (1993) Electrochemical reduction and oxidation of two cephalosporin antibiotics: Ceftriaxone (Rocephin) and cefoperazone (Cefobid). Electroanalysis (N. Y.), 5(7), 599-604.

[24] Ferreira, V.S., Zanoni, M.V.B. and Fogg, A.G. (1999) Cathodic stripping voltammetric determination of ceftazidime with reactive accumulation at a poly-L-lysine modified hanging mercury drop electrode. Analytia Chimica Acta, 384(2), 159-166.

[25] El-Obeid, H.A., Gad-Kariem, E.A., Al-Rashood, K.A., Al-Khames, H.A., El-Shafie, F.S. and Bawaseer, G.A.M. (1999) A selective colorimetric method for the determination of penicillins and cephalosporins with $\alpha$-aminoacyl functions. Analytical Letters, 32(14), 2809-2823.

[26] Mohamed, F.A. (1998) Spectrophotoemtric determination of sulphide and some sulphide producing compounds. In Proceedings of Assiut University 1st Pharmaceutical Science Conference, Faculty of Pharmacy, Assiut, 1998, 1-18.

[27] Metwally, F.H., Alwarthan, A.A. and Al-Tamimi, S.A. (2001) Flow-injection spectrophotometric determination of certain cephalosporins based on the formation of dyes. Il Farmaco, 56(8), 601-607.

[28] Sastry, C.S.P., Rao, S.G., Naidu, P.Y. and Srinivas, K.R. (1998) New spectrophotometric method for the determination of some drugs with iodine and wool fast blue BL. Talanta, 45(6), 1227-1234.

[29] Helaleh, M.I.H. and Abu-Nameh, E.S.M. (1998) A kinetic approach for determination of cefadroxil in pharmaceuticals by alkaline hydrolysis. Journal of $A O A C$ International, 81(3), 528-533.

[30] Ivama, V.M., Rodrigues, L.N.C., Guaratini, C.C.I. and Zanoni, M.V.B. (1999) Spectrophotometric determination of cefaclor in pharmaceutical preparations. Quimica Nova, 22(2), 201-204.

[31] Al-Momani, I.F. (2004) Flow-Injection spectrophotometric determination of amoxycillin, cefalexin, ampicillin, and cefradine in pharmaceutical formulations. Analytical Letters, 37(10), 2099-2110.

[32] Amin, A.S. and Shama, S.A. (2000) Vanadophosphoric acid as a modified reagent for the spectrophotometric determination of certain cephalosporins and their dosage forms. Monatshefte fur Chemie, 131(4), 313-319.

[33] Omai, K., Toyo'oka, T. and Miyano, H. (1984) Fluorigenic reagents for primary and secondary amines and thiols in high-performance liquid chromatography. A review. The Analyst (London), 109(11), 1365-1372.

[34] Olgun, N., Erturk, S. and Atmaca, S. (2002) Spectro- 
fluorimetric and spectrophotometric methods for the determination of vigabatrin in tablets. Journal of Pharmaceutical and Biomedical Analysis, 29(1-2), 1-5.

[35] Onal, A., Kepekci, S.E. and Oztunc, A. (2005) Spectrophotometric methods for the determination of antidepressant drug paroxetine hydrochloride in tablets. Journal of AOAC International, 88(2), 490-495.

[36] Olojo, R.O., Xia, R.H. and Abramson, J.J. (2005) Spectrophotometric and fluorometric assay of superoxide ion using 4-chloro-7-nitrobenzo-2-oxa-1,3-diazole. Analytical Biochemistry, 339(2), 338-344.

[37] El-Emam, A.A., Hansen, S.H., Moustafa, M.A., ElAshry, S.M. and El-Sherbiny, D.T. (2004) Determination of lisinopril in dosage forms and spiked human plasma through derivatization with 7-chloro-4-nitrobenzo-2-oxa1,3-diazole (NBD-Cl) followed by spectrophotometry or HPLC with fluorimetric detection. Journal of Pharmaceutical and Biomedical Analysis, 34(1), 35-44.

[38] Taha, E.A. (2003) Kinetic spectrophotometric methods for the determination of dothiepin hydrochloride in bulk and in drug formulation. Analytical and Bioanalytical Chemistry, 376(7), 1131-1136.

[39] Amin, A.S., Ragab, G.H. and Saleh, H. (2002) Colorimetric determination of $\beta$-blockers in pharmaceutical formulations. Journal of Pharmaceutical and Biomedical Analysis, 30(4), 1347-1353.

[40] Abdellatef, H.E. (2002) Kinetic spectrophotometric determination of tramadol hydrochloride in pharmaceutical formulation. Journal of Pharmaceutical and Biomedical Analysis, 29(5), 835-842.

[41] El-Enany, N., Belal F. and Rizk, M. (2004) Spectrophotometric determination of salbutamol in bulk and dosage forms after derivatization with 4 Chloro-7-nitrobenzo-2oxa-1,3-diazole (NBD Cl). Chemia Analityczna (Warsaw), 49(2), 261-269.

[42] Askal, H.F., Abdelmaged, O.H. and Kashaba, P.Y. (1995) Egyptian Journal of Analytical Chemistry, 4, 89-103.

[43] Alwarthan, A.A., Metwally, F.H. and Al-Tamimi, S.A. (1993) Spectrophotometric assay of certain cephalosporins based on formation of ethylene blue. Analytical Letters, 26(12), 2619-2635.

[44] Fogg, A.G. and Abdalla, M.A. (1985) Visible spectrophotometric determination of cephalosporins and penicillins by indophenol derivatisation with and without alkaline degradation to ammonia. Journal of Pharmaceutical and Biomedical Analysis, 3(4), 315-321.

[45] Abdalla, M.A., Fogg, A.G. and Burgess, C. (1982) Selective spectrophotometric determination of cephalosporins by alkaline degradation to hydrogen sulphide and formation of methylene blue. Analyst (London), 107(1273),
213-217.

[46] Topic Q2 (R1). (2005) Validation of analytical procedures: Text and methodology. International Conference on Harmonisation (ICH). http://www.emea.europa.eu/ pdfs/human/ich/038195en.pdf

[47] Heyden, Y.V., Nijhuis, A., Smeyers-Verbeke, J., Vandeginste, B.G.M. and Massart D.L. (2004) Guidance for rubustness/ruggedness test in method validation. Journal of Pharmaceutical and Biomedical Analysis, 24(5-6), 723753.

[48] Saleh, G.A., Askal, H., Darwish, I. and El-Shorbagi, A. (2003) Spectroscopic analytical study for the chargetransfer complexation of certain cephalosporins with chloranilic acid. Analytical Sciences, 19(2), 281-287.

[49] Harvey, D. (2000) Modern analytical chemistry. McGrawHill, Boston, MA.

[50] Fogg, A.G., Fayad, N.M., Burgess, C. and McGlynn, A. (1979) Differential pulse polarographic determination of cephalosporins and their degradation products. Analytica Chimica Acta, 108, 205-211.

[51] Fogg, A.G., Fayad, N.M. and Burgess, C. (1979) Differential pulse polarographic study of the degradation of cephalexin: Determination of hydrogen sulphide and other degradation products. Analytica Chimica Acta, 110 (1), 107-115.

[52] Fogg, A.G. and Martin, M.J. (1981) Differential pulse polarographic determination of degradation products of cephalosporins: Comparison of the degradation of cephaloglycin in neutral solution with that of cephalexin. Analyst (London), 106(1268), 1213-1217.

[53] Fogg, A.G., Abdalla, M.A. and Henriques, H.P. (1982) Titrimetric determination of the yield of sulphide formed by alkaline degradation of cephalosporins. Analyst (London), 107(1273), 449-452.

[54] Abdalla, M.A., Fogg, A.G., Baber, J.G. and Burgess, C. (1983) Air-segmented continuous-flow visible spectrophotometric determination of cephalosporins in drug formulations by alkaline degradation to hydrogen sulphide and formation of methylene blue and determination of sulphide-producing impurities including cephalosporins in penicillin samples. Analyst (London), 108(1282), 53-57.

[55] Grekas, N. and Calokerinos, A.C. (1990) Continuous flow molecular emission cavity analysis of cephalosporins by alkaline degradation to sulphide. Analyst (London), 115(5), 613-616.

[56] Svehla, G. (1979) Vogel's textbook of macro and semimicro qualitative inorganic analysis. 5th Edition, The Chaucer Press, Great Britain. 